\title{
THE PRESENCE OF A SYSTEMATIC ERROR IN SDO/HMI DATA
}

\author{
G.V. Rudenko \\ Institute of Solar-Terrestrial Physics SB RAS, \\ Irkutsk,Russia,rud@iszf.irk.ru
}

\author{
I.S. Dmitrienko \\ Institute of Solar-Terrestrial Physics SB RAS, \\ Irkutsk,Russia,dmitrien@iszf.irk.ru
}

\begin{abstract}
In this paper, we came to the conclusion that there is a systematic error in SDO/HMI (Helioseismic and Magnetic Imager aboard the Solar Dynamics Observatory) vector magnetic data, which reveals itself in a deviation from the radial direction of the knot magnetic fields manifesting themselves on magnetograms in the form of small grains in a strong magnetic field. This deviation demonstrates a dependence on the distance to
\end{abstract}

the disk center, which cannot be a property of the magnetic field - it can only be artificially introduced into the data. We suggest a simple method for correcting vector magnetograms, which eliminates the detected systematic error. grams.

Keywords: Sun, magnetic field, vector magneto-

\section{INTRODUCTION}

Vector magnetic data from the SDO/HMI spacecraft represent a significant breakthrough in solar magnetography. Spatial resolution, quality of full disk vector magnetograms, regularity and high duty cadence of observations are unparalleled either in ground or in satellite measurements. Also invaluable is the contribution that can be made in the near future by an everexpanding time series of continuous observations to space weather predictions and basic research into the magnetic nature of solar activity. In particular, we can expect a significant improvement in the reliability of the forecast of parameters of the solar wind and interplanetary magnetic field (IMF), specifically its polarity in near-Earth space due to the possibility of using new vector synoptic maps [Gusain et al., 2013].

Only synoptic maps of the longitudinal field have been used for this purpose before. The advantage of the new vector synoptic maps is due to two factors. First, vector measurements allow us to extrapolate the field over the boundary field radial component (Neumann problem). From a physical point of view, this extrapolation is more valid than the extrapolation over the longitudinal component because real measurements are carried out at a level where not only potential but also more general force-free approximation is inapplicable.

Under these conditions, different boundary-value problems should inevitably give different extrapolation results, which in particular will also yield different radial field components at the boundary. Second, we construct a synoptic map of $B_{\mathrm{r}}$ as a scalar value (unlike maps of the longitudinal magnetic component $B_{\mathrm{los}}$ ). This is an important point since mapping of the synoptic distribution for a nonscalar value is not quite correct.

For any large project that makes new physical information widely available, it is important to remove any significant errors of artificial or natural origin, if there are such errors and they can be detected, before using this information or at the initial stage of the project. Our paper addresses this very kind of problem for new SDO/HMI data. We establish that there is a significant systematic error in the data provided. We clearly find this error by analyzing measurements of knot magnetic fields concentrating in nodes of the network of convection cells of the quiet Sun. Knots appear on magnetograms in the form of fine grains of a strong magnetic field and result from raking of magnetic tubes by horizontal motions of the surrounding plasma. This raking leads to a concentration of magnetic flux and subsequent radialization of the field. The compactness property of knot fields and the significant excess of background values (>500 G) make it possible to identify them using a fairly simple algorithm. The radiality property inherent to knot fields is used as the main criterion for testing magnetic field measurements. Section 1 shows that any vector SDO/HMI magnetogram exhibits the same systematic deviation (up to $20^{\circ}$ ) of the knot field from the radial direction toward the limb, which depends on the distance to the solar disk center. Since the observational result should not depend on the position of an observer, we conclude that this dependence can have only artificial cause, which cannot be eliminated by modern technologies for obtaining and processing Stokes parameters, used to derive final values of the vector magnetic field. In Section 2, we propose correcting original vector magnetograms, proceeding from the assumption that the systematic deviation is in fact independent of the measurement point on the solar disk and is caused by an error in measuring the field inclination angle to the line of sight. Effects of this error in knot fields lead to its apparent dependence on the position on the solar disk. We show that our correction almost eliminates the effects of unnatural behavior of knot fields.

\section{IDENTIFICATION AND ANALYSIS OF KNOT FIELDS}

\subsection{Selection and geometric interpretation of knot magnetic fields}

Our analysis rests on the natural assumption of the radial character of isolated small-scale magnetic struc- 
tures with large amplitudes. Most of these structures represent knot magnetic fields, which concentrate in nodes of the network of convective cells of the quiet Sun. Identification of the structures of interest on magnetograms and their physical characteristics can be described by the following selection algorithm (SA):

- using the LABEL_REGION IDL procedure, chose a complete set of isolated regions with $|\mathbf{B}|>300 \mathrm{G}$;

- take from the set only $A_{n}$ regions with less than 35 pixels and $\max \left|\mathbf{B}\left(A_{n}\right)\right|>500 \mathrm{G}$;

- find $\left(i_{\max }^{n}, i_{\max }^{n}\right):\left|\mathbf{B}\left(i_{\max }^{n}, i_{\max }^{n}\right)\right|=\max \left|\mathbf{B}\left(A_{n}\right)\right|$ pixel for each $A_{n}$;

- assign magnetic field values $B^{n}=\mathbf{B}\left(i_{\max }^{n}, i_{\max }^{n}\right)$ and position radius vector $r^{n}=r\left(i_{\max }^{n}, i_{\max }^{n}\right)$ to each $A_{n}$.

The $\mathbf{B}_{n}$ and $r_{n}$ values are used for further analysis. In the following, for simplicity, we omit the index $n$.

Figure 1 gives a typical example of determining positions of centers of knot regions (red crosses). The isolated knot regions are fairly evenly distributed throughout the region of the quiet Sun, concentrating largely near active regions (green areas).

To estimate the degree of radiality of the knot regions, we use three knot parameters $\frac{\left|B_{\mathrm{r}}^{\text {los }}\right|}{|\mathbf{B}|}, \alpha$, and $\beta$, where

$$
\frac{B_{\mathrm{r}}^{\operatorname{los}}}{|\mathbf{B}|}=\frac{B_{\mathrm{los}}}{\cos \mu} /|\mathbf{B}|,
$$

$B_{\text {los }}=B_{z}$ is the projection of vector $\mathbf{B}$ along the line of sight; $\mu$ is the angle between the line of sight and the radius vector of the point position on the disk $\left(\left|B_{\mathrm{r}}^{\text {los }}\right|=\left|B_{\mathrm{r}}\right|=|\mathbf{B}|\right.$ if the field is strictly radial); $\alpha$ is the angle between the radius vector of the observation point and the magnetic field component in the meridian plane given by the radius vector of the observation point and the $\mathrm{Z}$ axis of the line of sight ( $\alpha>0$ in the direction to the limb, $\alpha=0$ if the field is strictly radial); $\beta$ is the angle between the field line vector (we always assume that it is directed from the Sun) and the meridian plane ( $\beta=0$ if the field is strictly radial). The geometry of the $\alpha$ and $\beta$ angles is shown in Figure 2 .

It is important to note that no information is needed about the azimuthal field direction to derive $\left|B_{\mathrm{r}}^{\text {los }}\right| /|\mathbf{B}|$, whereas deriving the angular knot parameters $\alpha$ and $\beta$ requires procedure for eliminating the uncertainty of the $\Phi$ azimuth (unavoidable in measurements of the uncertainty of the transverse field). In this paper, to eliminate the uncertainty, we adopt the method described in [Rudenko, Anfinogentov, 2014].

\subsection{Distribution of HMI knot characteristics over the disk}

We have examined the knot parameters that characterize the deviation of knot fields from radiality for a sufficiently large set of 30 SDO/HMI magnetograms arbitrarily selected over a period 2012-2014. In all the

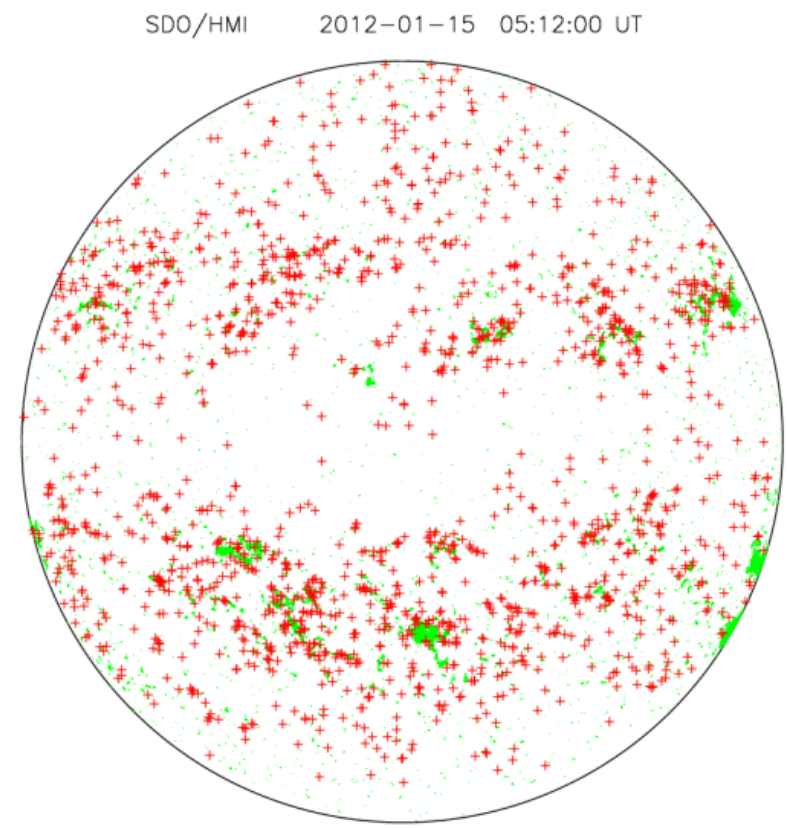

Figure 1.SDO/HMI 2012-01-15 05:12:00 UT. Typical distribution of magnetic knots over the solar disk: $|\mathbf{B}|>300 \mathrm{G}$ (green areas), knots (red crosses)

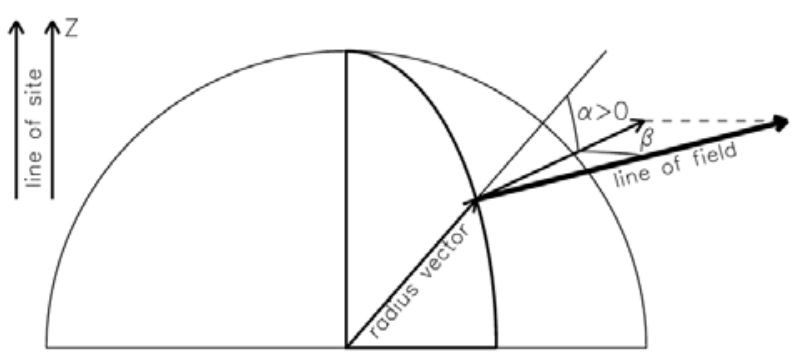

Figure 2. Angular characteristics of the deviation of the magnetic field vector from radiality

magnetograms, the $\frac{\left|B_{\mathrm{r}}^{\text {los }}\right|}{|\mathbf{B}|}$, $\alpha$, and $\beta$ parameters displayed almost identical behavior on the solar disk (Figure 1).

Figure 3 indicates that only the $\beta$ parameter shows the expected (in the absence of artifacts in the data) statistical behavior with an average zero value.

The remaining $\frac{\left|B_{\mathrm{r}}^{\text {los }}\right|}{|\mathbf{B}|}$ and $\alpha$ parameters demonstrate a clear dependence of their average values on the distance from the center of the solar disk. On the one hand, nonzero $\frac{\left|B_{\mathrm{r}}^{\operatorname{los}}\right|}{|\mathbf{B}|}$ and $\alpha$ contradict the hypothesis on radiality of knot fields. On the other hand, their dependence on the distance to the center of the solar disk indicates the presence of an artificial error. Indeed, even if we exclude the radiality property from the chosen magnetic elements, there would be in principle no statistical relationship between the knot parameters and the distance to the solar disk center. The same magnetic elements cannot give different values of the field, depending on their apparent position on the solar disk. It is also clear that the 

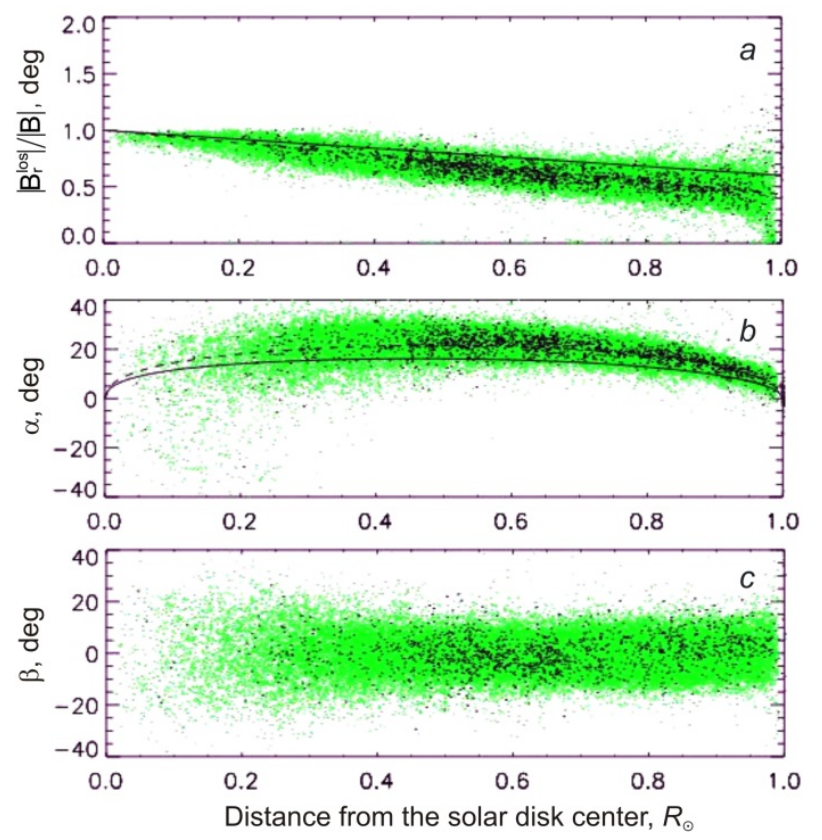

Figure 3. Parameters of knot elements versus the distance to the solar disk center: $a-\left|B_{\mathrm{r}}^{\operatorname{los}}\right| /|\mathbf{B}| ; b-\alpha, c-\beta$ for the complete set of magnetograms (green areas) and for points of one SDO/HMI 2012-01-15 05:12:00 UT magnetogram (black dots)

the error we detected cannot arise from the subsequent processing of initial magnetograms associated with the method for solving the $\pi$-uncertainty problem of the transverse magnetic component of measurements. In the presence of the error generated only by the method for solving the $\pi$-uncertainty problem, we would not observe any statistical relationship of $\frac{\left|B_{\mathrm{r}}^{\text {los }}\right|}{|\mathbf{B}|}$ with distance, which does not depend on the method for solving the $\pi$ problem. Notice that the maximum deviation of $\alpha$ of the order of $20^{\circ}$ at mean distances and an almost twofold decrease in the ratio $\frac{\left|B_{\mathrm{r}}^{\text {los }}\right|}{|\mathbf{B}|}$ near the limb point to a significant artificial error.

Note the apparent simplicity of the relationship of $\frac{\left|B_{\mathrm{r}}^{\text {los }}\right|}{|\mathbf{B}|}$ with distance; this relationship in Figure 3, $a$ is visually perceived as being close to linear. Write it in the form

$$
\frac{\left|B_{\mathrm{r}}^{\text {los }}\right|}{|\mathbf{B}|}=1-k r_{\perp}
$$

wherer is the distance from the disk center in solar radii. From the complete set of magnetograms, we have obtained a fitting value of the linear coefficient in Formula (2) equal to

$$
k=k_{\mathrm{fit}}=0.565 \text {. }
$$

The corresponding dependence is shown in Figure 3, $a$ by the dashed line (the solid line in Figure 3, $a$ and lines in Figure 3, $b$ will be discussed later in the text).
Note that the results presented in Figure 3 and (2) with coefficient (3) explain well Figure 4, $b$ from [Leka et al., 2017], which shows the $\left|B_{\mathrm{r}}^{\text {los }}\right|$ dependence on $\left|B_{\mathrm{r}}\right|$ in the polar region. The statistical distribution of these values exhibits a clear, nearly twofold decrease in the former value relative to the latter (the same as in our case for $r \sim 1$ (Figure 3, a).

Thus, the presence of a significant systematic error in HMI/SDO data can be considered a well-established fact.

\section{SYSTEMATIC ERROR CORRECTION}

The very simple dependence of the knot parameter $\frac{\left|B_{\mathrm{r}}^{\text {los }}\right|}{|\mathbf{B}|}$

on distance $r$ (Figure 3, a) gives rise to the possi-

bility of finding a correction method that can eliminate, at least formally, the observed statistical effects. Initially, we can suggest two approaches to the solution of this problem: the geometric approach - the correction depends on the position of a measured element on the disk; the local approach - the correction depends only on measured values themselves and does not depend on the position of a measured element on the disk. The former approach proved to be rather problematic. We at least could not find an appropriate way of its implementation. Our correction, based on the second assumption, is quite simple and yields preliminary results which we consider quite reasonable. We hope that the new magnetograms that have no these shortcomings as compared to original ones may be more appropriate for further practical use both for developing the global corona model [Riley et al., 2006, 2014] and for nonlinear modeling of active magnetic regions [Sun et al. 2012; Thalmann et al., 2012; Tadesse et al., 2013].

\subsection{Method}

Introduce the following notations: $\left|\mathbf{B}^{*}\right|$ is the modulus of the true (desired) magnetic field; $\gamma^{*}$ is the inclination of the true magnetic field; $\Phi^{*}$ is the azimuth of the true transverse magnetic field; $B_{\text {los }}^{*}=|\mathbf{B}| \cos \gamma^{*}$ is the longitudinal component of the true magnetic field.

Refer the corresponding notations without asterisks to initially measured field values.

Assume the following identities to be true for measurements at each point of the disk:

$$
\begin{aligned}
& \Phi^{*} \equiv \Phi, \\
& B_{\mathrm{los}}^{*}=\left|\mathbf{B}^{*}\right| \cos \gamma^{*} \equiv B_{\mathrm{los}}=|\mathbf{B}| \cos \gamma .
\end{aligned}
$$

Suppose that the true field is radial. In this case, in Formula (2) for knots, we have

$r=\sin \gamma^{*}$,

$\cos \mu=\cos \gamma^{*}$.

Using (1), (6), (7), Formula (2) can be rewritten in the following form:

$$
\frac{\left|B_{\mathrm{r}}^{\operatorname{los}}\right|}{|\mathbf{B}|}=\frac{|\mathbf{B}| \cos \gamma}{\cos \gamma^{*}|\mathbf{B}|}=\frac{\cos \gamma}{\cos \gamma^{*}}=1-k \sin \gamma^{*} .
$$


Equation (8) gives a unique relationship between the true and measured values of the inclination. The $\gamma^{*}$ value can be found using the Newton algorithm. After finding $\gamma^{*}$ from (5) and (7), determine the true field modulus

$$
\left|\mathbf{B}^{*}\right|=|\mathbf{B}| \frac{\cos \gamma}{\cos \gamma^{*}}=|\mathbf{B}|\left(1-k \sin \gamma^{*}\right) .
$$

Formulas (4), (8), (9) allow us to uniquely determine the derived values of $\left|\mathbf{B}^{*}\right|, \gamma^{*}$, and $\Phi^{*}$ through their initial values and $k$. Extending these relationships to all points of the solar disk, we obtain a magnetogram with new values of the inclination and modulus of the field with preservation of the longitudinal component of the field and the azimuth of the transverse field. In this case, the average value of $\frac{\left|B_{\mathrm{r}}^{* \text { los }}\right|}{\left|\mathbf{B}^{*}\right|}$ in knot regions should take on a value of 1 everywhere. It is important to note that the correction based on Formulas (4), (8), (9) does not depend on the procedure for eliminating the $\pi$-uncertainty of the $\Phi$ azimuth of the transverse field and can be made before this procedure.

\subsection{Distribution of knot parameters for a corrected magnetogram}

Let us first consider the result of correction with the value of $k$ from (3) (Figure 4) for the knot regions previously isolated on uncorrected magnetograms. As expected, the distance dependence of the knot parameter $\frac{\left|B_{\mathrm{r}}^{\text {los }}\right|}{|\mathbf{B}|}$ is quite well removed.

Only variance of this quantity depends on the distance to the center of the solar disk. Furthermore, the dependence of the second knot parameter $\alpha$ on the distance to the solar disk center also disappears. Indeed, according to the definition of $\alpha$, from (2) we can predict its behavior on the disk (before correction), setting $\beta=0$ :

$$
\alpha=\arccos \left(\left(1-k r_{\perp}\right)\left(1-r_{\perp}^{2}\right)-r_{\perp} \sqrt{1-\left(1-k r_{\perp}\right)^{2}\left(1-r_{\perp}^{2}\right)}\right) .
$$

Dependence (10) is shown in Figure $3, b$ by the dashed line for $k=0.565$. It is clear that with the disappearance of dependence (2), the average $\alpha$ should be zero everywhere.

The example given in Figure 4 is not entirely correct, despite the noticeable improvement in knot characteristics. To select knot regions, in the SA procedure we use uncorrected ("incorrect") values of the field modulus, which, as we have seen, changes significantly. The knot parameters obtained from the corrected magnetogram for $k=0.565$ yield a result with some residual statistical dependence on the distance near the limb (Figure 5).

This result is quite natural since the $k$ value obtained from the selection was determined from the set of "incorrect" magnetograms. As a result of the selection, we have found the most appropriate value of $k=0.4$ (the solid line in Figure 3,a). Figure 6 indicates that the correction with the $k$ value found practically eliminates the dependence of knot parameters on the distance to the solar disk center. Dependence (10) corresponding to this $k$ is shown by the solid line in Figure $3, b$. Naturally, the latter is somewhat offset from the mean values of the knot parameter $\alpha$.
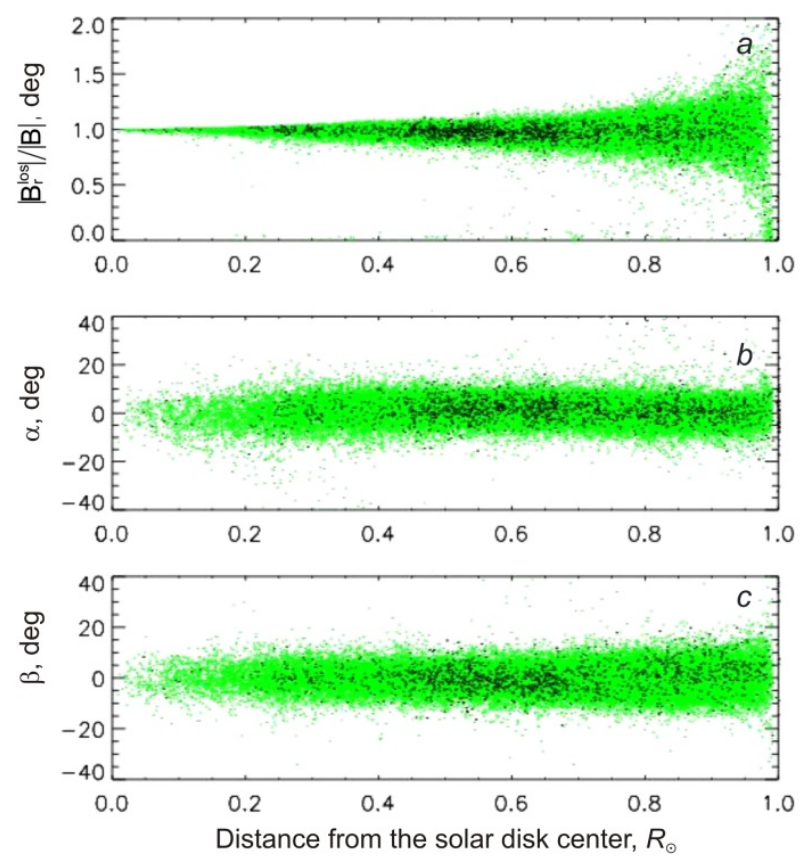

Figure 4. Corrected dependence of knot parameters on the distance to the solar disk center $(k=0.565)$ : $a$ $\left|B_{\mathrm{r}}^{\operatorname{los}}\right| /|\mathbf{B}| ; b-\alpha, c-\beta$ for the complete set of magnetograms (green areas) and for points of one SDO/HMI 201201-15 05:12:00 UT magnetogram (black dots). SA uses an uncorrected magnetogram
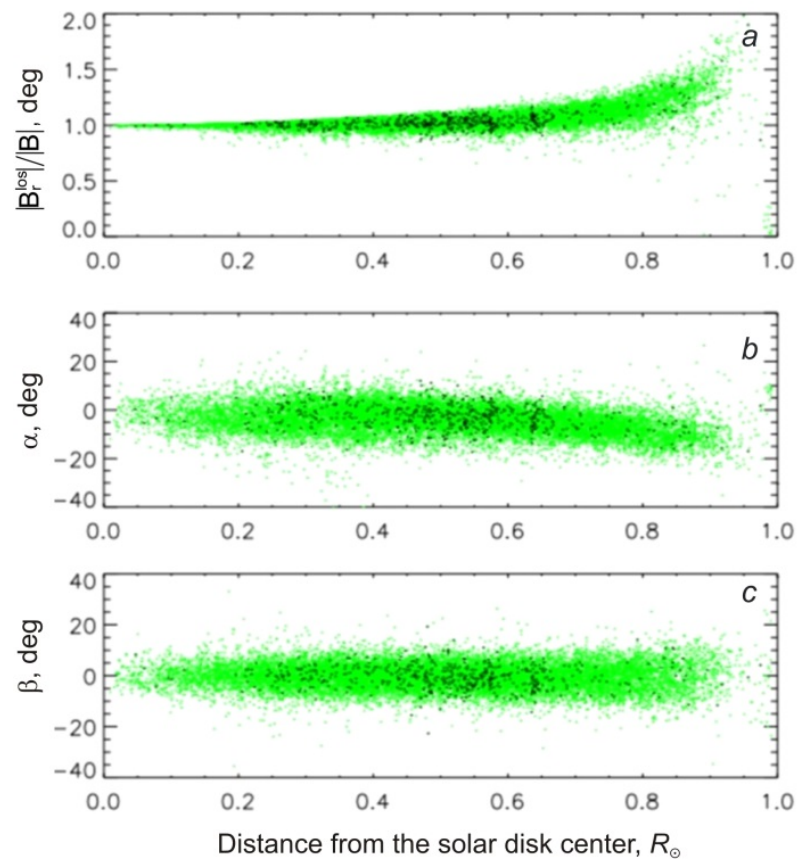

Figure 5. Corrected dependence of knot parameters on the distance to the solar disk center ( $k=0.565): a-\left|B_{\mathrm{r}}^{\text {los }}\right| /|\mathbf{B}|$; $b-\alpha, c-\beta$ for the complete set of magnetograms (green areas) and for points of one SDO/HMI 2012-01-15 05:12:00 UT magnetogram (black dots). SA uses a corrected magnetogram 
So, we have magnetograms knot fields on which correspond to the natural assumption of radiality. At any point of the magnetogram, inclination and modulus changes depend only on the local value of $\gamma$ according to Formulas (8), (9) for $k=0.4$. These changes appreciably alter inclinations and strength of the magnetic field (Figure 7).

Appropriateness of this correction for magnetic quantities in active regions can be estimated later only from results of applied solutions of physical problems because in the general case we have no assumptions about the true orientation of the magnetic field.
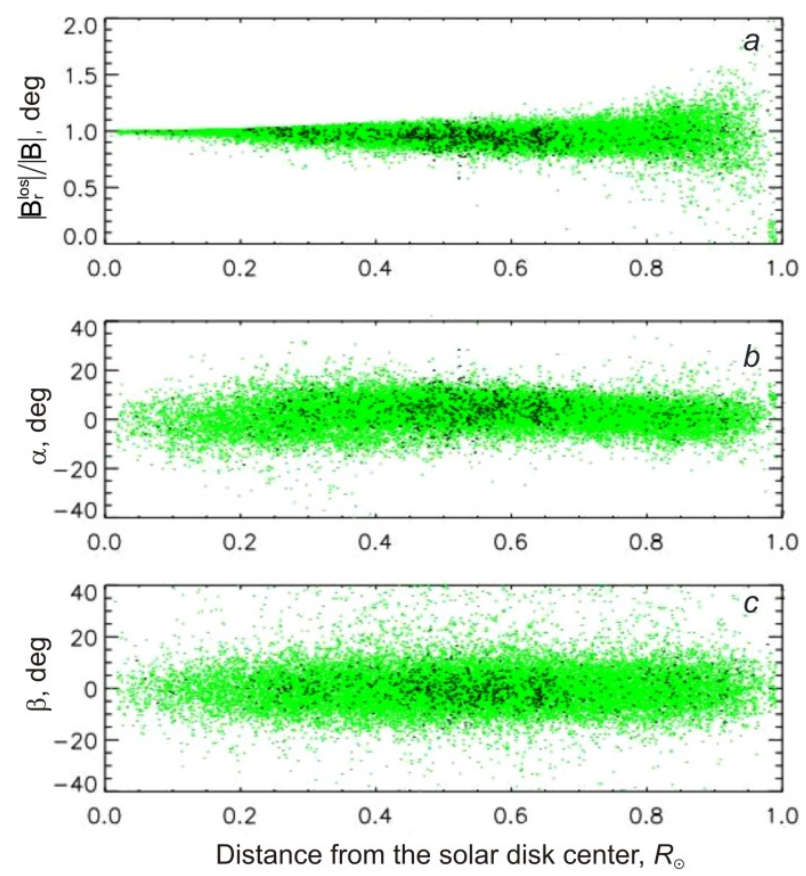

Figure 6. Corrected dependence of knot parameters on the distance to the solar disk center $(k=0.5): a-\left|B_{\mathrm{r}}^{\text {los }}\right| /|\mathbf{B}|$; $b-\alpha, c-\beta$ for the complete set of magnetograms (green areas) and for points of one SDO/HMI 2012-01-15 05:12:00 UT magnetogram (black dots). SA uses a corrected magnetogram
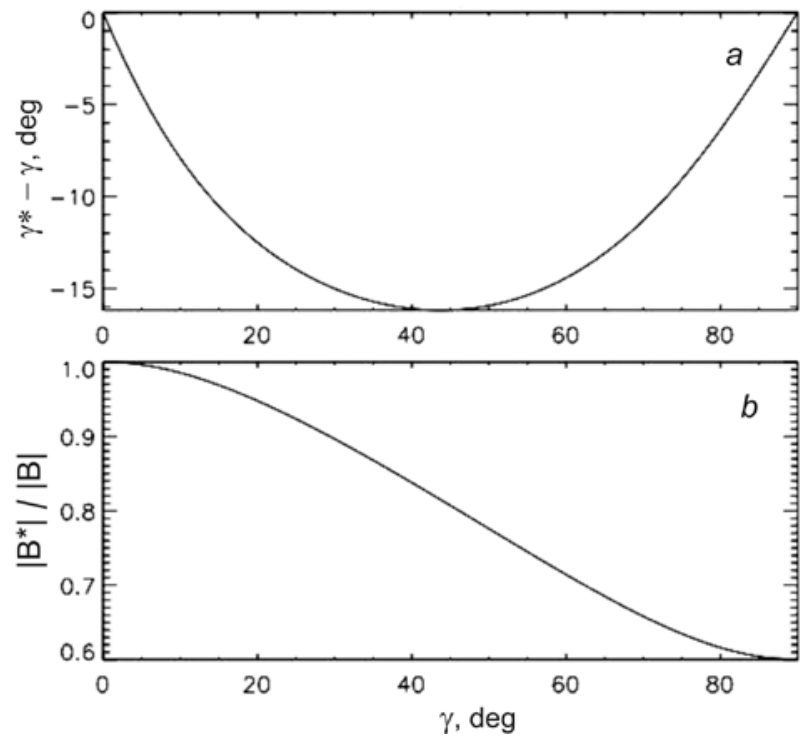

Figure 7. Changes in the inclination (a) and modulus of the field $(b)$ after correction

\section{CONCLUSION}

We have shown that the SDO/HMI vector magnetic data provided by the SDO Helioseismic and Magnetic Imager have a significant error. It manifests itself in the fact that the magnetic field in small-scale magnetic elements with high field strength (magnetic knots) deviates from the radial direction toward the solar limb. The magnitude of this deviation depends on the distance to the center of the visible solar disk and reaches a maximum of $\sim 20^{\circ}$ at distances of about $0.4 R$ from the center of the disk.

We have proposed a correction that eliminates this systematic error. This correction is tentative and requires further testing in specific applied problems. It may be used to find causes of the systematic error in data and to eliminate this error on the hardware level.

\section{REFERENCES}

Gusain S., Pevtsov A.A., Rudenko G.V., Anfinogentov S.A. First synoptic maps of photospheric vector magnetic field from SOLIS/VSM: Non-radial magnetic fields and hemispheric pattern of helicity. Astrophys. J. 2013, vol. 772, no. 1, pp. 52. DOI: 10.1088/0004-637X/772/1/52.

Leka K.D. Barnes G., Wagner E.L. Radial magnetic field component from line-of-sight magnetograms // Solar Phys. 2017, vol. 292, iss. 2, article id.no. 36, p. 26. DOI: 10.1007/s11207-017-1057-8.

Riley P., Linker J.A., Mikíc Z., Lionello R., Ledvina S.A., Luhmann J.G. A comparison between global solar magnetohydrodynamic and potential field source surface model results // Astrophys. J. 2006, vol. 653, pp. 1510. DOI: 10.1086/508565.

Riley P., Ben-Nun M., Linker J.A., Mikic Z., Svalgaard L., Harvey J., Bertello L., Hoeksema T., Liu Y., Ulrich R. A multiobservatory inter-comparison of line-of-sight synoptic solar magnetograms.Solar Phys. 2014, vol. 289, pp. 769. DOI: 10.1007/s11207-013-0353-1.

Rudenko G.V., Anfinogentov S.A. Very fast and accurate azimuth disambiguation of vector magnetograms.Solar Phys. 2014, vol. 289, pp. 1499-1516. DOI: 10.1007/s11207-0130437-y.

Sun X., Hoeksema J.T., Liu Y., Wiegelmann T., Hayashi K., Chen Q., Thalmann J. Evolution of magnetic field and energy in a major eruptive active region based on SDO/HMI observation. Astrophys. J. 2012, vol. 748, pp. 77. DOI: $10.1088 / 0004-$ 637X/748/2/77.

Tadesse T., Wiegelmann T., Inhester B., MacNeice P., Pevtsov A., Sun X. Full-disk nonlinear force-free field extrapolation of SDO/HMI and SOLIS/VSM magnetograms. Astron. Astrophys. 2013. vol. 550. pp. A14. DOI: 10.1051/00046361/201220044.

Thalmann J.K., Pietarila A., Sun X., Wiegelmann T. Nonlinear force-free field modeling of a solar active region using SDO/HMI and SOLIS/VSM data. Astrophys. J. 2012, vol. 144,

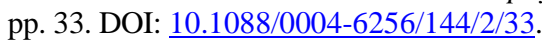

How to cite this article

Rudenko G.V., Dmitrienko I.S.The presence of a systematic error in SDO/HMI data.Solar-Terrestrial Physics. 2018, vol. 4, no. 2, pp. 3-7. DOI: $10.12737 /$ stp-42201801 